13. Europäischer Kongress für Trauma und Notfallchirurgie, ESTES, 2012 in Basel

\title{
Basel welcomes Europe
}

Dominik Heim ${ }^{a}$, Reto Babst ${ }^{b}$

a PD Dr. med., Chefarzt Chirurgie, Spital Frutigen

b Prof. Dr. med., Chefarzt Unfallchirurgie, Departementsleiter Chirurgie, Luzerner Kantonsspital
1 Die Schweizer Hausarztmedizin am Wonca Europe Kongress 2009 in Basel. Schweiz Ärztezeitung. 2010;91(8):297-8.

2 Die Schweizerische Gesellschaft für Traumatologie und Versicherungsmedizin SGTV - eine interdisziplinäre Gesellschaft mit europäischer Vernetzung. Schweiz Ärztezeitung. 2008;89(16): 696-7.

Korrespondenz:

PD Dr. med. Dominik Heim

Spital Frutigen

CH-3714 Frutigen

dominik.heim@spitalfmi.ch
Es ist aussergewöhnlich, auf diesem Weg «Reklame» für einen Kongress zu machen - es ist auch aussergewöhnlich, dass eine grosse europäische, medizinische Gesellschaft ihren Kongress in der Schweiz abhält. 2009 war dies der Fall mit dem europäischen Wonca Europe Kongress [1]. 2012 wird nun vom 12. bis 15. Mai im Kongresszentrum in Basel der 13. Europäische Kongress für Trauma und Notfallchirurgie der ESTES (European Society for Trauma and Emergency Surgery) stattfinden. Mitorganisiert wird dieser chirurgische Grossanlass von der Schweizerischen Gesellschaft für Traumatologie und Versicherungsmedizin, SGTV, und von der Schweizerischen Gesellschaft für Allgemeinchirurgie und Traumatologie, SGACT. Dass die Schweiz den Zuschlag für diesen Anlass erhalten hat, hängt auch mit dem 100. Geburtstag der SGTV zusammen, die am 7. Januar 1912 in Bern als Gesellschaft Schweizer Unfallärzte in Bern gegründet wurde [2].

\section{Dachorganisation von $\mathbf{2 6}$ Gesellschaften}

ESTES ist das Produkt einer Fusion der European Trauma Society (ETS) mit der European Association for Trauma and Emergency Surgery (EATES) im Jahr 2007 [2]. ESTES ist heute die Dachorganisation von 26 nationalen unfall- und notfallchirurgischen Gesellschaften in Europa mit mehr als 10000 institutionellen Mitgliedern. Das wissenschaftliche Spektrum ist entsprechend dem Fachgebiet sehr breit, es reicht von der «Fussfraktur über die akute Cholecystitis bis zum Schädel-Hirn-Trauma». Damit entspricht das Spektrum demjenigen eines Allgemeinchirurgen, der zur Zeit wieder - im Rahmen der Diskussion um den «Acute care surgeon» - im intensiven Gespräch ist.

In einer Zeit, in der es auf der ganzen Welt aus geographischen, ökonomischen oder andern Gründen immer noch auch kleinere Spitäler mit ihren spezifischen Bedürfnissen gibt, ist die Funktion dieses Kongresses, in einem breiten Spektrum ausgebildete und tätige Chirurgen und Orthopäden, Notfallmediziner, Operationsschwestern und Sanitäter zu informieren und weiterzubilden. Studenten werden in der Absicht angesprochen, einen entsprechenden Nachwuchs an Acute care surgeons «heranzubilden».

Instructional lecture courses, Keynote lectures mit anschliessenden freien Mittteilungen, Guest Symposia (Efort, SGC, SGOT, Küntscher Kreis, Efost, EBJIS, AOTrauma und SOFCOT), Workshops und Satellite Symposia der Industrie werden durchgeführt werden. Neu in Basel sind die Curtain-up sessions, die speziellen Institutionen in Basel (Universität Basel), in der Schweiz (Internationales Komitee vom Roten

\section{$13^{\text {th }}$ European Congress of}

Trauma \& Emergency Surgery

Many ways - one goal

May $12-15,2012 \cdot$ Basel / Switzerland

Organised by

European Society for Trauma \& Emergency Surgery

In cooperation with

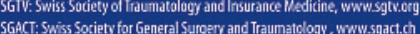

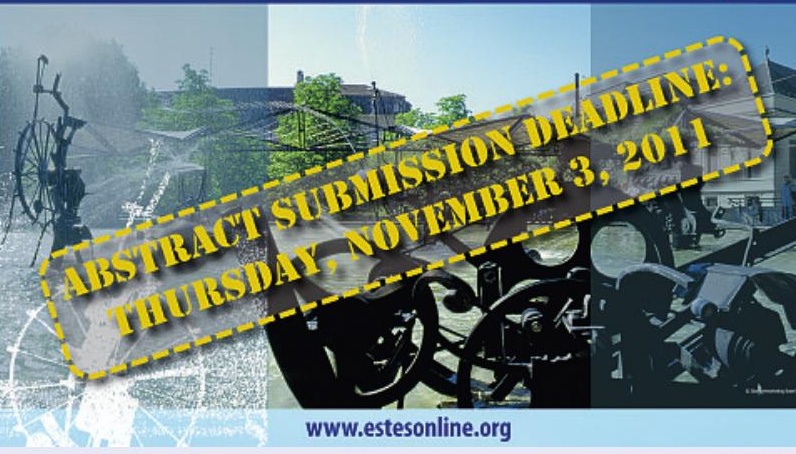

ECTES 2012: Die Einreichefrist für Abstracts dauert noch bis zum November 2011

Kreuz) und in der Welt (Trauma in Asien) gewidmet sein werden. «How-I-do-it-Sessions» am frühen Morgen werden operative Aspekte beleuchten, und in den «12-to-12-Sessions» am Mittag werden ganz pragmatische Kurzvorlesungen einzelne Aspekte des Traumas und der Notfallchirurgie behandeln. Eine spezielle Zusammenarbeit erhält der ESTES-Kongress dieses Jahr auch in der Teilnahme der AO (Arbeitsgemeinschaft für Osteosynthesefragen), die 1958 in Biel gegründet wurde und unterdessen das grösste traumatologische Netzwerke in der Welt darstellt. Die 16 ausgewählten Main topics (www.estesonline.org, www.sgtv.org und www.sgact.ch) versprechen spannende Referate, animierte Diskussionen und informative Kongressmomente.

\section{Lokale Akzente in den Social events}

Ein Kongress ist auch in die Kongress-Stadt, in die Kongress-Region und in das Kongress-Land eingebettet. Die Basler Bevölkerung wird am BAZ Forum am 11. Mai 2012 mit wissenschaftlichen Vorträgen über den Kongress informiert werden, eine öffentliche Sitzung für die Bevölkerung wird am 15.Mai im Kongresszentrum stattfinden, und die lokalen Medien sind eingeladen, den Kongress zu begleiten. In den Social events werden typisch schweizerische und lokale Eigenheiten berücksichtigt. Ein spezieller Akzent wurde auf die Darstellung der schweizerischen Musikszene in all ihren Facetten gelegt: Eliana Burki und iAlpinisti werden den Kongress eröffnen, George Gruntz und Max Lässer werden am Swiss evening im Joggeli-Fussballstadium auftreten, und eine Basler Clique wird den Kongress musikalisch abschliessen. Dieter Ammann und Band aus Zofingen werden am President's dinner im Tinguely Museum für ein Verschmelzen von moderner, bewegter Kunst und bewegtem Jazz sorgen.

Basel soll den Kongressteilnehmern als ein medizinischer Anlass in Erinnerung bleiben, bei dem sich europäische Medizin und Schweizer Kultur zu einem Rendez-vous am Rheinknie getroffen haben. 